\title{
Sampling of explosive residues: the use of a gelatine-based medium for the recovery of ammonium nitrate
}

\author{
Amaral, M. A. ${ }^{1,2,3, *}$, Yasin, S. ${ }^{4}$, Gibson, A. P. ${ }^{3,5}$, Morgan, R. M. ${ }^{1,2}$ \\ ${ }^{1}$ Department of Security and Crime Science, University College London, 35 Tavistock Square, London WC1H 9EZ, United Kingdom \\ ${ }^{2}$ Centre for the Forensic Sciences, University College London, 35 Tavistock Square, London WC1H 9EZ, United Kingdom \\ ${ }^{3}$ Department of Medical Physics \& Biomedical Engineering, University College London, Gower Street, London WC1E 6BT, United Kingdom \\ ${ }^{4}$ School of Applied Science, London South Bank University, 103 Borough Road, London SE1 0AA, United Kingdom \\ ${ }^{5}$ Institute for Sustainable Heritage, University College London, Central House, London WC1E 0NN, United Kingdom \\ ${ }^{*}$ Corresponding author \\ E-mail address: mark.amaral.14@ucl.ac.uk
}

\begin{abstract}
Forensic scientists must be able to recover traces of any original explosive materials not consumed in the detonation, in a careful controlled manner to aid a crime reconstruction. In current sampling techniques, the collection efficiency of post-blast residue is highly variable and often dependent on the swabbing materials and solvent systems used. To address these method limitations, this study presents a gelatine-based sampling medium and assesses its capabilities for the collection of ammonium nitrate. Common surfaces were spotted with a known concentration of ammonium nitrate, the unset gel applied, allowed to set, and then peeled from the surface. The gel was dissolved, and solid phase extraction employed to isolate the target explosive compound and remove the constituents of the gel. The eluate was concentrated and subsequently analysed and quantified. Overall, the gel formulation was able to collect ammonium nitrate from all of the test surfaces, with recoveries ranging from $0.1 \%$ to $61.7 \%$. This study presents a gelatine-based formulation that has the potential to become a valuable asset in the forensic tool kit for the collection of explosive traces. A key attribute of the gel is that it offers an alternative recovery tool to conventional swabbing and solvent extraction methods.
\end{abstract}

\section{Keywords}

Post-blast analysis; Trace evidence; Evidence recovery; Gelatine

\section{Introduction}

The use of explosive materials by individuals and groups to propagate terror poses a significant threat to public safety, political stability and the global economy [1]. When explosives have been detonated, the residual explosive material deposited at a crime scene is of significant value in forensic investigations and subsequent crime reconstructions. Recovery of these traces can assist investigators in determining the type of explosive compound(s) used, identifying potential sources of improvised explosive device components, comparing physical evidence from the scene to a potential suspect, 
corroborating statements and potentially linking criminal cases [2]. However, the majority of explosive residues consist of relatively simple anions, cations and vapours which have little diagnostic value [35]. Therefore, the primary goal of the forensic scientist is to recover traces of the original explosive materials that were not consumed in the detonation [6].

\subsection{Explosives}

Chemical explosives can be differentiated from one another based on their explosive behaviour, which is characterised by their molecular structure [7]. They can be divided into three classes based on their relative sensitivities: primary, secondary and tertiary (Table 1). Primary explosives are the most sensitive and undergo a rapid transition from burning to detonation and are able to propagate a detonation wave to less sensitive explosives. Unlike primary explosives, secondary explosives are relatively insensitive to shock, friction, flame or heat and are initiated by a strong explosive shock wave that is produced by the explosion of a primary explosive. Upon detonation, the compounds of secondary explosives will dissociate instantaneously [8]. Tertiary explosives are insensitive to shock and therefore the most stable. They require a secondary explosive, functioning as a booster, to cause detonation [9].

\section{Table 1}

The three classes of chemical explosives based on their relative sensitivities and some examples of the explosive compounds in each class.

\begin{tabular}{cc}
\hline Class & Examples \\
\hline Primary & Lead azide, lead styphnate, mercury fulminate and some organic peroxides \\
Secondary & Research Department Explosive (RDX), 2,4,6-trinitrotoluene (TNT) and \\
Tertiary & pentaerythritol tetranitrate (PETN) \\
& Ammonium nitrate fuel oil (ANFO) \\
\hline
\end{tabular}

Explosives can also be classified based on their provenance: military, commercial or home-made [9]. Unlike military and commercial explosives, home-made explosives do not have a legitimate use and recently, there has been an increase in the use of organic peroxides in explosive attacks [10]. This development in recent explosive material selections demonstrates the dynamic shift in criminal choices as explosion detection capabilities of security forces becomes enhanced. This trend is exemplified in the changing nature of materials used in terrorist attacks that have occurred over the last quartercentury [10-13]. 


\subsection{Explosive Residues}

Scientific literature pertaining to the origin and distribution of explosive residues is limited. It has been posited that explosive residues are derived from the partial reaction of the thin outer layer of an explosive charge. This results from the reflection of the shock wave from the surface back into the reaction zone of the device [14]. Whilst the exact mechanism by which these traces persist during detonation events is unknown, it is accepted that they do, and can subsequently be found at post-blast scenes. Explosives undergoing deflagration (rapid burning) as opposed to detonation are termed low explosives and leave large deposits of unconsumed particulates which are easier to identify by investigators $[6,15]$. Residue recoveries from explosives undergoing detonation are much more challenging [15]; in such events, undetonated explosive residues are still present as a result of the incomplete combustion of the explosive [6]. These trace residues are likely to be present in low amounts, potentially at the nanogram level, and therefore extreme care needs to be taken in their recovery and analysis [16].

\subsection{Recovery}

Residual traces of explosives can persist for long periods of time depending on the material upon which they are adsorbed or deposited. The collection method used to recover post-blast residues depends on a number of factors including the physical form of the residue, the type of deposition surface, and whether a low explosive or high explosive was used [15]. Mechanical vacuuming or physical sweeping of the epicentre of the explosion is considered to be the most efficient manner of collecting the unconsumed particulates of low explosives, whereas swabbing and solvent washes are frequently employed in the recovery of residues from high explosives [15,17].

The collection efficiency of post-blast residue based on current sampling techniques is highly variable, between approximately 10 and 90 per cent, and is often dependent on the swabbing medium and solvent used [18]. Certain solvents such as acetone can damage certain substrates resulting in interfering background material being collected which can reduce recovery efficiency [19]. Also affecting the recovery efficiency is the unavoidable delay that exists between the time of collection and subsequent extraction and analysis. During this time period there is a decline in the concentration of volatile explosives as well as an increase in the formation of breakdown products [18]. Cotton swabs moistened with an ethanol and water mixture are commonly used for the collection of both inorganic and organic explosive residues $[18,20]$. Due to the low recoveries obtained from porous surfaces using traditional swabbing methods, vacuum sampling is also employed [21]. 
In recent years, the increased use of high-energy improvised explosive materials, such as organic peroxides and homemade mixtures of inorganic and organic explosive compounds, in terrorist attacks has been observed around the world [20,22]. As such, there is a demand for an optimised sampling procedure capable of recovering a wide array of post-blast residues that may be encountered at these scenes. In addition, a single-step extraction method for both organic and inorganic compounds is required as traditional sequential extraction methods, which make use of multiple solvents with differing polarities, results in the loss of certain compounds [22], which may have significance for the interpretation of this form of intelligence or evidence in the investigation context.

\subsection{Hydrogels}

Hydrogels have primarily been used in the medical and pharmaceutical sectors; however, recent studies have shown hydrogels, are being designed and tailored for a variety of different applications [23]. Previous research into the use of hydrogels in the recovery of particulates and forensic sampling has been conducted, including the incorporation of hydrogels in a testing kit for TNT [24] and the use of hydrogels for the collection of amino acids and DNA from fingermarks [25]. Hydrogels are comprised of a cross-linked polymeric network. Due to the presence of hydrophilic functional groups attached to the polymer backbone, hydrogels have the ability to absorb large quantities of water within the spaces available among the polymeric chains [26,27]. Water absorbed by the hydrogel allows for the free diffusion of solute molecules [26]. The amount of water absorbed by the hydrogel, or total water holding capacity, is dictated by the cross-linking density [28]. As cross-linking density increases, there is a decrease in equilibrium swelling as a result of decrease in the hydrophilic groups [27]. Physical properties, such as diffusivity of the entrapped molecules and mechanical strength, are dictated by the mesh size of the polymeric network [29].

\subsection{Aim}

The purpose of this study was to create and then evaluate the use of a gelatine-based medium for the collection of trace particulate explosive residues. The versatility of a gel medium means it can be used on a wide array of surfaces and has the potential to provide a new sample collection technique that can be employed by forensic investigators at post-blast scenes to overcome the limitations of conventional collection techniques. Therefore, the study sought to:

1. Create a gelatine-based collection medium to provide an easy application method, requiring little to no scientific knowledge or technical expertise; 
2. Assess the degree to which this gelatine-based collection medium could offer a more efficient sampling technique, resulting in better collection efficiencies than the current methods being employed.

\section{Materials and Methods}

The gelatine-based collection medium constituents (gelatine powder, arrowroot powder and glycerine) and test surfaces used were all sourced from local vendors. The inorganic explosive, ammonium nitrate (99.0 \% purity), along with the solvents used, were acquired from Sigma-Aldrich (Gillingham, Dorset, U.K.); all solvents were HPLC grade unless otherwise stated. Bromelain tablets (500 mg), used to prevent the gelatine from resetting, were purchased from Holland \& Barrett (Nuneaton, Warwickshire, U.K.). Oasis ${ }^{\circledR}$ MCX solid-phase extraction (SPE) cartridges (30 $\mu \mathrm{m}$ copolymer reverse-phase $60 \mathrm{mg}$ / $20 \mathrm{~mL}$ ) from Waters (Elstee, Herts, U.K.) were used for the extraction and isolation of ammonium nitrate from the gel matrix.

\subsection{Gel Formulation}

The individual components of the gel formulation are readily obtainable from shops and do not have to be acquired through chemical suppliers, affording a cost-effective sampling medium. The gel was formulated by combining gelatine powder, arrowroot powder, glycerine, and water $\left(\mathrm{H}_{2} \mathrm{O}\right)$ in a mass ratio of 3:1:1:5. Using a hotplate with stirrer, gelatine powder was added to $\mathrm{H}_{2} \mathrm{O}$ in a beaker and heated to $75^{\circ} \mathrm{C}$ until dissolved, stirring continually. Once dissolved, the arrowroot powder was added followed by the glycerine. The mixture was then kept at $75^{\circ} \mathrm{C}$ for 5 minutes, stirring continually.

\subsection{Recovery Surfaces}

A total of six different surfaces, cut into $2 \mathrm{~cm} \mathrm{x} 2 \mathrm{~cm}$ squares, were used in this study: copper, acrylonitrile butadiene styrene (ABS, chosen to represent plastics), glass, carpet, a cotton polyester blend fabric, and unfinished wood (Fig. 1.) to represent common surfaces where explosive particulate recovery is often required. Each surface was cleaned with methanol $(\mathrm{MeOH})$ prior to analysis to ensure they were free from contamination. 
Copper

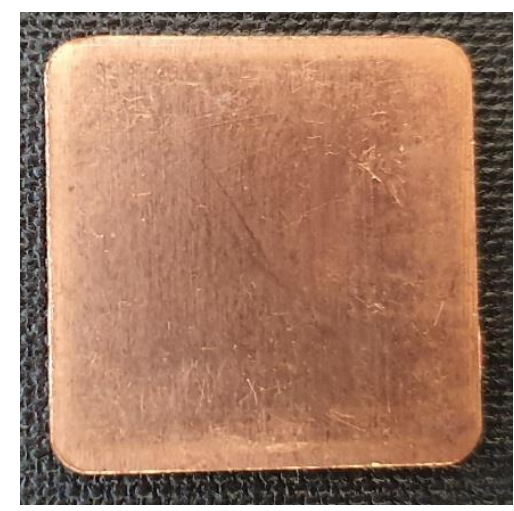

Carpet

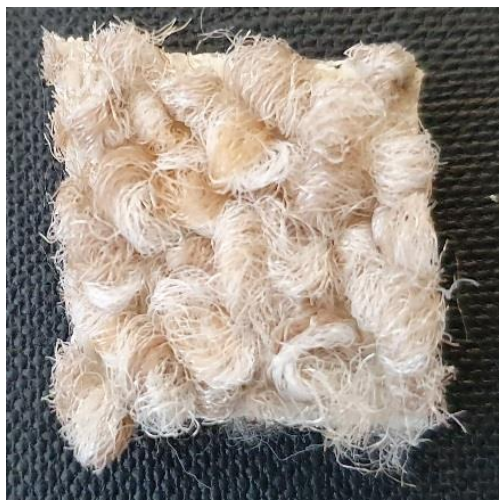

ABS

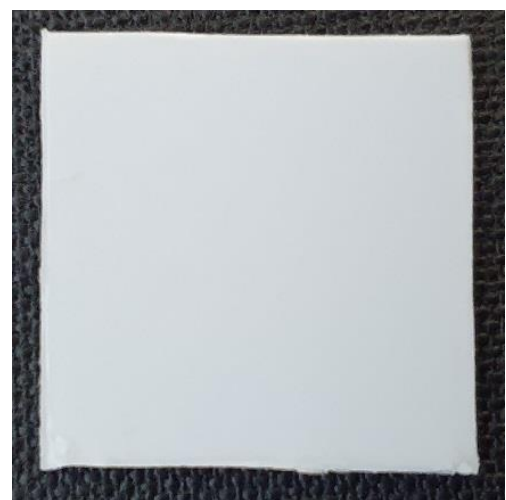

Fabric

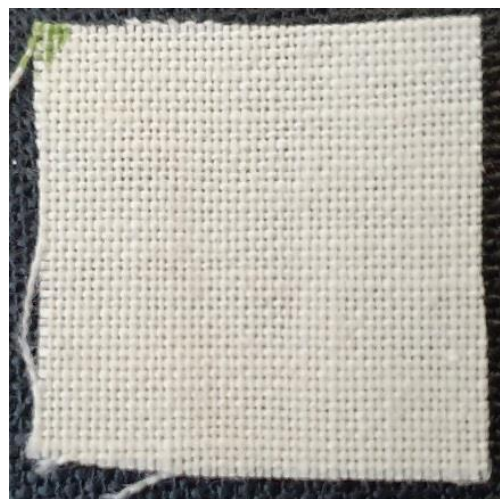

Glass

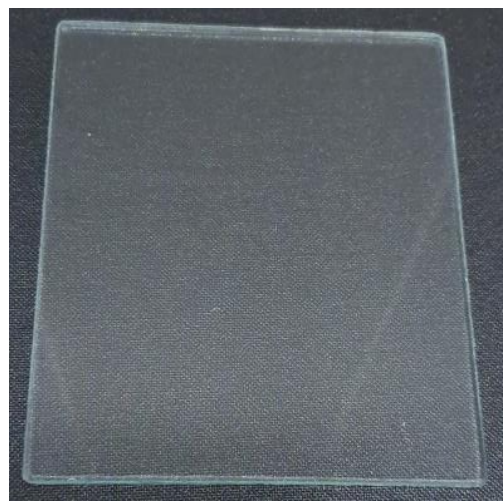

Wood

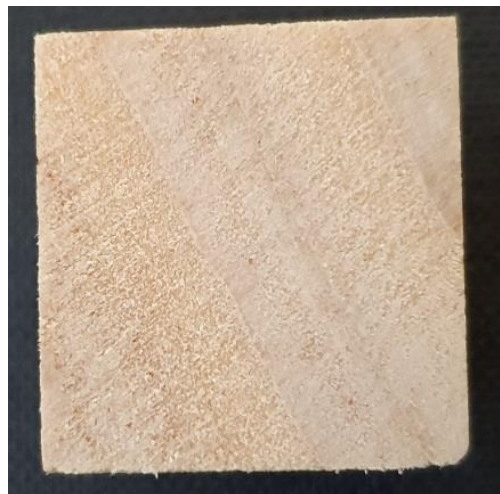

Fig. 1. Non-porous (top) and porous (bottom) surfaces used in this study.

\subsection{Ammonium Nitrate}

Ammonium nitrate has been used as an explosive in numerous terrorist incidents [12,30,31]. It was selected as the target analyte for this study due to its relative safety and stability, as well as the ease of use and availability of the compound. A $1,000 \mathrm{mg} / \mathrm{L}$ working solution of ammonium nitrate was prepared by weighing $0.1 \mathrm{~g}$ of ammonium nitrate in a $100 \mathrm{~cm}^{3}$ volumetric flask and diluting to volume with $\mathrm{MeOH}$. Using a pipette, $100 \mu \mathrm{L}$ of ammonium nitrate stock solution was spotted onto the centre of each of the surfaces (five replicates of each test surface) and allowed to evaporate in order to recrystallise the explosive from the solvent.

\subsection{Application}

Using a disposable syringe, $2 \mathrm{~cm}^{3}$ of gel was applied to each surface (each surface was analysed in quintuplicate; $\mathrm{n}=5$ for each of the six surfaces) and left to solidify for 10 minutes. Once solidified, the gel was peeled from the surface with forceps and placed into a $10 \mathrm{~cm}^{3}$ glass vial. The vial was then placed onto a hotplate, $5 \mathrm{~mL}$ of $\mathrm{H}_{2} \mathrm{O}$ added, and heated at $75^{\circ} \mathrm{C}$ until dissolved. In order to prevent the gel from solidifying, bromelain (an enzyme found in the stems of pineapples) was added to each sample ( $0.2 \mathrm{~g}$ of crushed (powdered) bromelain for every $6 \mathrm{~g}$ of gelatine used). During method development, 
bromelain did not impact the chromatographic profile obtained when added to solutions of ammonium solution.

\subsection{Extraction}

Following dissolution of the samples, SPE was employed as a sample clean-up technique to isolate the analytes of interest from the gelatine-based matrix. The SPE cartridges were conditioned with $5 \mathrm{~mL}$ of $\mathrm{MeOH}$, and then equilibrated with $10 \mathrm{~mL} \mathrm{H}_{2} \mathrm{O}$. The samples $(\mathrm{v}=5 \mathrm{~mL})$ were then loaded onto the cartridges and allowed to elute under vacuum. The cartridges were then washed with $5 \mathrm{~mL}$ of $\mathrm{H}_{2} \mathrm{O}$ and dried under vacuum. Finally, $5 \mathrm{~mL}$ of acetonitrile $(\mathrm{ACN})$ was added to the cartridges and the eluates collected. The eluates were dried under a stream of nitrogen gas and reconstituted in $1 \mathrm{~mL}$ of $\mathrm{MeOH}$ prior to analysis. The absolute recovery of the SPE method was assessed by loading $1 \mathrm{~mL}$ of a 1,000 $\mathrm{mg} / \mathrm{L}$ ammonium nitration solution directly onto the cartridge and the eluate quantified. The absolute recovery with respect to ammonium nitrate was $92 \% \pm 5 \%$ (mean \pm standard deviation, $\mathrm{n}=5$ ).

\subsection{Analysis}

A Prominence UFLC (Ultra Fast Liquid Chromatograph) system from Shimadzu fitted with an Acclaim $^{\mathrm{TM}}$ Explosive E2 column was used to analyse the eluates. The method used (Table 2) was adapted from the United States Environmental Protection Agency (EPA) Method 8330B for nitroaromatics, nitramines, and nitrate esters [32]. Although the recommended wavelength for the detector was $230 \mathrm{~nm}$, during the method development stage, it was found that setting the detector to $214 \mathrm{~nm}$ yielded a greater response for ammonium nitrate. As such, $214 \mathrm{~nm}$ was selected as the detector wavelength. Using this method, the retention time of ammonium nitrate was determined to be 1.8 minutes.

\section{Table 2}

Liquid chromatography (LC) operating conditions for the analysis of ammonium nitrate.

\begin{tabular}{cc}
\hline Analytical Conditions & Method \\
\hline Column & Acclaim $^{\mathrm{TM}}$ Explosive E2 $(3.0 \times 150 \mathrm{~mm}, 3 \mu \mathrm{m}, 120 \AA)$ \\
Flow rate & $0.3250 \mathrm{~mL} / \mathrm{min}$ \\
Eluent & $48 / 52 \mathrm{v} / \mathrm{v} \mathrm{MeOH} / \mathrm{H}_{2} \mathrm{O}$ \\
Gradient & Isocratic \\
Temperature & $30{ }^{\circ} \mathrm{C}$ \\
Detection & $\mathrm{UV} 214 \mathrm{~nm}$ \\
\hline
\end{tabular}




\subsection{Quantification}

In order to quantify any ammonium nitrate recovered, a nine-point calibration curve was established $(1 \mathrm{mg} / \mathrm{L}$ to $1,000 \mathrm{mg} / \mathrm{L})$. The average peak area was plotted against the concentration of each calibration standard (run in triplicate) injected onto the column and the linearity evaluated using the $\mathrm{R}^{2}$ coefficient of determination. The $\mathrm{R}^{2}$ value indicated that a good reliability existed in the linear relationships between the analyte concentrations and the peak areas (Fig. 2.). Once the calibration curve had been constructed and the linear range determined, two calibration checks were performed to assess the reliability of the calibration curve in quantifying ammonium nitrate. This was carried out using two independently prepared solutions at concentrations of 250 and $850 \mathrm{mg} / \mathrm{L}$. The accuracy, with respect to determining the true concentration of ammonium nitrate in a sample, was determined to be $99.5 \%$ and $99.2 \%$ respectively.

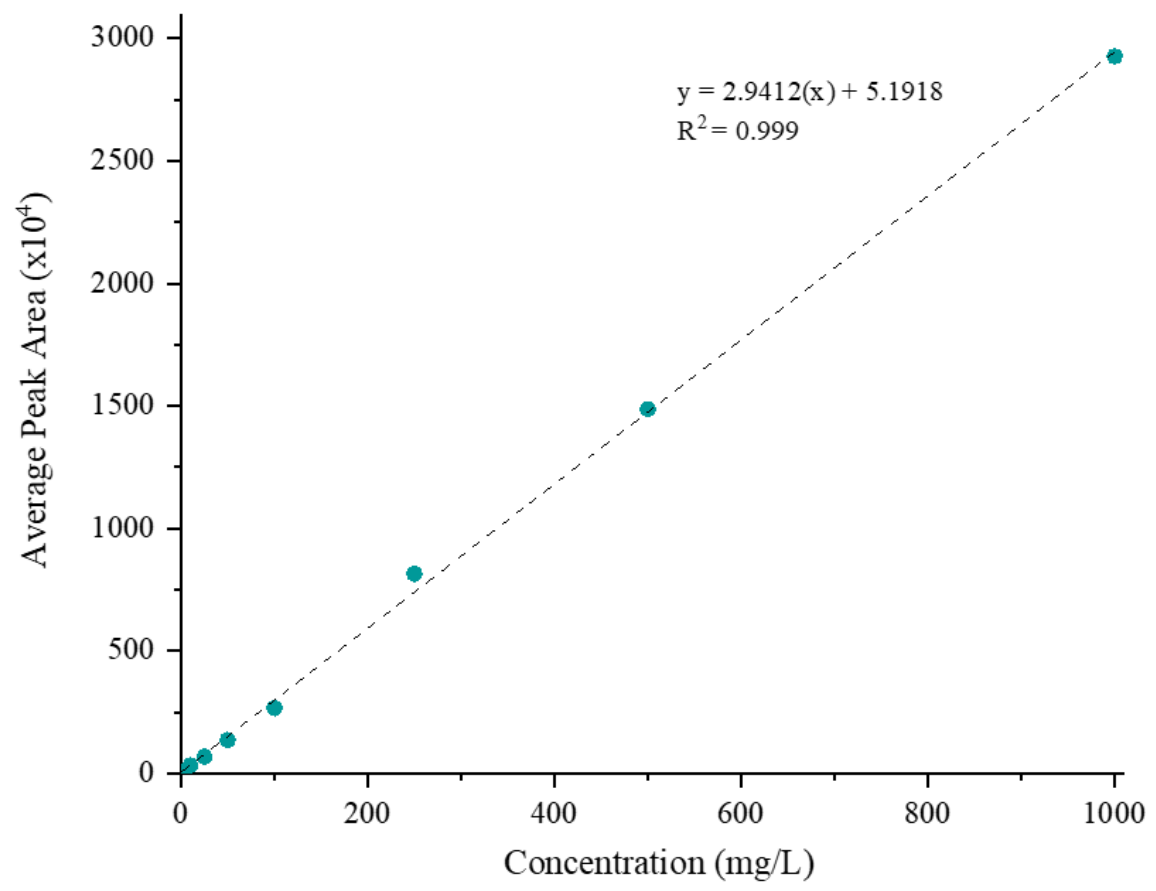

Fig. 2. LC calibration curve of ammonium nitrate (reference standard).

\subsection{Negative Controls}

A series of negative controls was carried out to ensure that the response obtained from analysis was a direct result of ammonium nitrate being detected and not a background contaminate present on the surfaces used, or a constituent of the gel. To assess this, the procedure outlined above was repeated for each of the six different surface types which were not spotted with ammonium nitrate. Additionally, the gel formulation was analysed separately, having not been applied to a surface. 


\subsection{Statistics}

A two-sample $t$-Test assuming unequal variances was employed to assess whether the mean recoveries obtained from porous and non-porous surfaces differed significantly $(\mathrm{p}<0.05)$. A one-way ANOVA was conducted to compare the recovery of ammonium nitrate across the three different porous surfaces in order to assess if the means significantly $(\mathrm{p}<0.05)$ differed. This was repeated for non-porous surfaces.

\section{Results and Discussion}

\subsection{Negative Controls}

No response at 1.8 minutes was measured in any of the negative controls. As such, the peak obtained in the recovery samples was a direct result of ammonium nitrate being detected in eluates and not resulting from a background contaminate present on the test surfaces or in the gel formulation.

\subsection{Porous Surfaces}

Recoveries from the porous surfaces ranged from $0.1 \%$ to $14.9 \%$, with the highest recoveries obtained from carpet (average recovery of 6.8\%) and the lowest recoveries from untreated wood (average recovery of $1.1 \%$ ) (Table 3). The recoveries obtained from the three porous surfaces are graphically represented in Fig. 3. When comparing the recoveries of ammonium nitrate obtained from wood, fabric and carpet, the results did not statistically differ $(\mathrm{p}>0.05)$.

\section{Table 3}

Gel recovery results of ammonium nitrate from three porous surfaces. Units of concentration are $\mathrm{mg} / \mathrm{L}$.

\begin{tabular}{|c|c|c|c|c|}
\hline Surface & $\begin{array}{c}\text { Ammonium } \\
\text { Nitrate Conc. }\end{array}$ & $\begin{array}{c}\text { Avg. Conc. } \pm \text { SD } \\
(\% \mathrm{CV})\end{array}$ & Recovery (\%) & $\begin{array}{c}\text { Avg. } \\
\text { Recovery }(\%)\end{array}$ \\
\hline \multirow{5}{*}{ Wood } & 13.5 & & 1.4 & \multirow{5}{*}{1.1} \\
\hline & 6.0 & $10.7 \pm 7.3$ & 0.6 & \\
\hline & 22.7 & $(68.2 \%)$ & 2.3 & \\
\hline & 1.8 & & 0.1 & \\
\hline & 10.0 & & 1.0 & \\
\hline \multirow{5}{*}{ Fabric } & 11.2 & & 1.1 & \multirow{5}{*}{1.6} \\
\hline & 5.0 & & 0.5 & \\
\hline & 30.4 & $16.1 \pm 8.7$ & 3.0 & \\
\hline & 20.4 & $(53.7 \%)$ & 2.0 & \\
\hline & 13.7 & & 1.4 & \\
\hline \multirow{5}{*}{ Carpet } & 3.8 & \multirow{5}{*}{$\begin{array}{c}67.8 \pm 55.9 \\
(82.5 \%)\end{array}$} & 0.4 & \multirow{5}{*}{6.8} \\
\hline & 22.9 & & 2.3 & \\
\hline & 46.0 & & 4.6 & \\
\hline & 149.3 & & 14.9 & \\
\hline & 117.0 & & 11.7 & \\
\hline
\end{tabular}




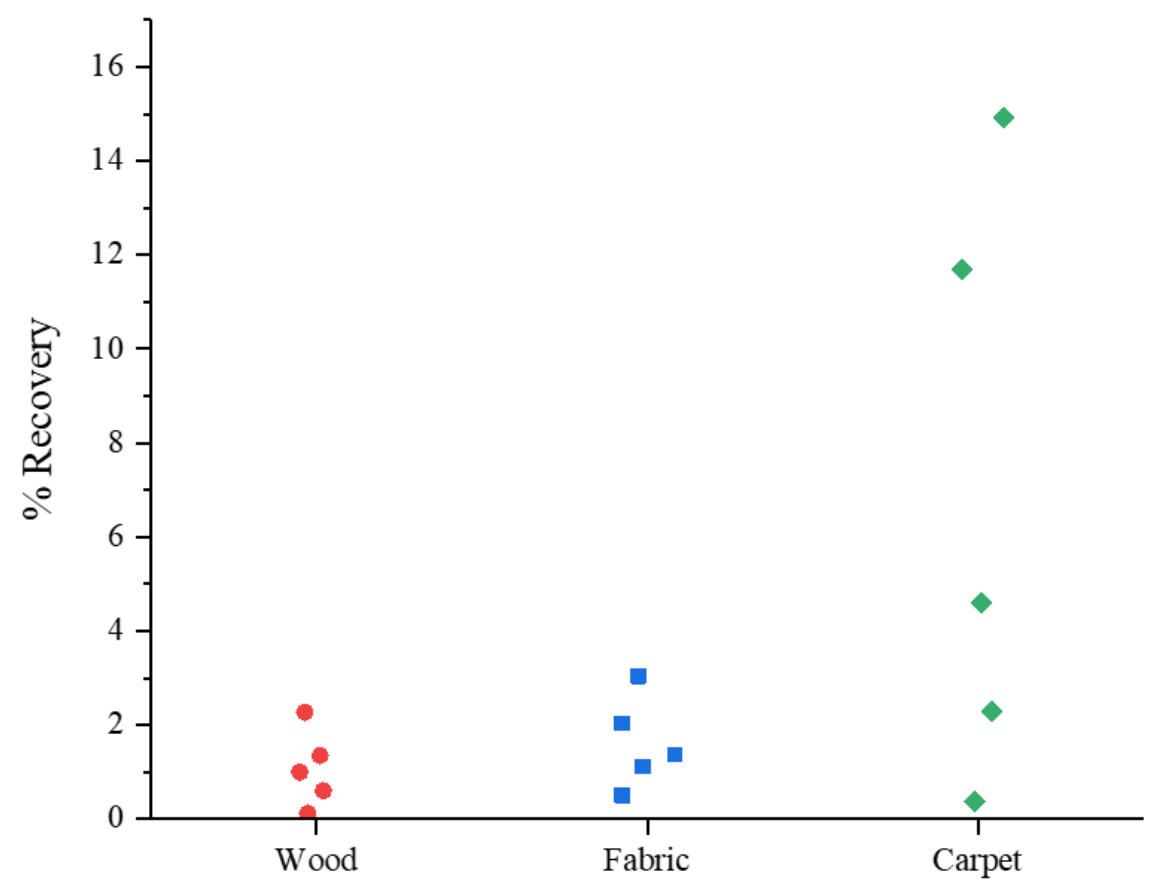

Fig. 3. Scatter plot of the recoveries $(\%)$ obtained from the three porous surfaces tested.

\subsection{Non-porous Surfaces}

Recoveries from non-porous surfaces ranged from $10.0 \%$ to $61.6 \%$, with the highest recoveries obtained from ABS (average recovery of 38.6\%) and the lowest recoveries from copper (average recovery of $18.4 \%$ ) (Table 4). The recoveries obtained from the three non-porous surfaces are graphically represented in Fig. 4. When comparing the recoveries of ammonium nitrate obtained from copper, glass, and ABS, the results did not statistically differ $(\mathrm{p}>0.05)$. 


\section{Table 4}

Gel recovery results of ammonium nitrate from three non-porous surfaces. Units of concentration are $\mathrm{mg} / \mathrm{L}$.

\begin{tabular}{|c|c|c|c|c|}
\hline Surface & $\begin{array}{c}\text { Ammonium } \\
\text { Nitrate Conc. }\end{array}$ & $\begin{array}{c}\text { Avg. Conc. } \pm \text { SD } \\
(\% \mathrm{CV})\end{array}$ & Recovery (\%) & $\begin{array}{c}\text { Avg. } \\
\text { Recovery }(\%)\end{array}$ \\
\hline \multirow{5}{*}{ Copper } & 166.9 & \multirow{5}{*}{$\begin{array}{c}184.1 \pm 56.4 \\
(30.6 \%)\end{array}$} & 16.7 & \multirow{5}{*}{18.4} \\
\hline & 100.3 & & 10.0 & \\
\hline & 217.2 & & 21.7 & \\
\hline & 167.3 & & 16.7 & \\
\hline & 268.8 & & 26.9 & \\
\hline \multirow{5}{*}{ Glass } & 101.3 & \multirow{5}{*}{$\begin{array}{c}240.9 \pm 93.2 \\
\quad(38.7 \%)\end{array}$} & 10.1 & \multirow{5}{*}{24.1} \\
\hline & 359.7 & & 36.0 & \\
\hline & 180.9 & & 18.1 & \\
\hline & 319.9 & & 32.0 & \\
\hline & 242.5 & & 24.3 & \\
\hline \multirow{5}{*}{ ABS } & 459.0 & \multirow{5}{*}{$\begin{array}{c}385.8 \pm 188.0 \\
(48.8 \%)\end{array}$} & 45.9 & \multirow{5}{*}{38.6} \\
\hline & 163.5 & & 16.4 & \\
\hline & 526.5 & & 52.7 & \\
\hline & 616.0 & & 61.6 & \\
\hline & 163.9 & & 16.4 & \\
\hline
\end{tabular}

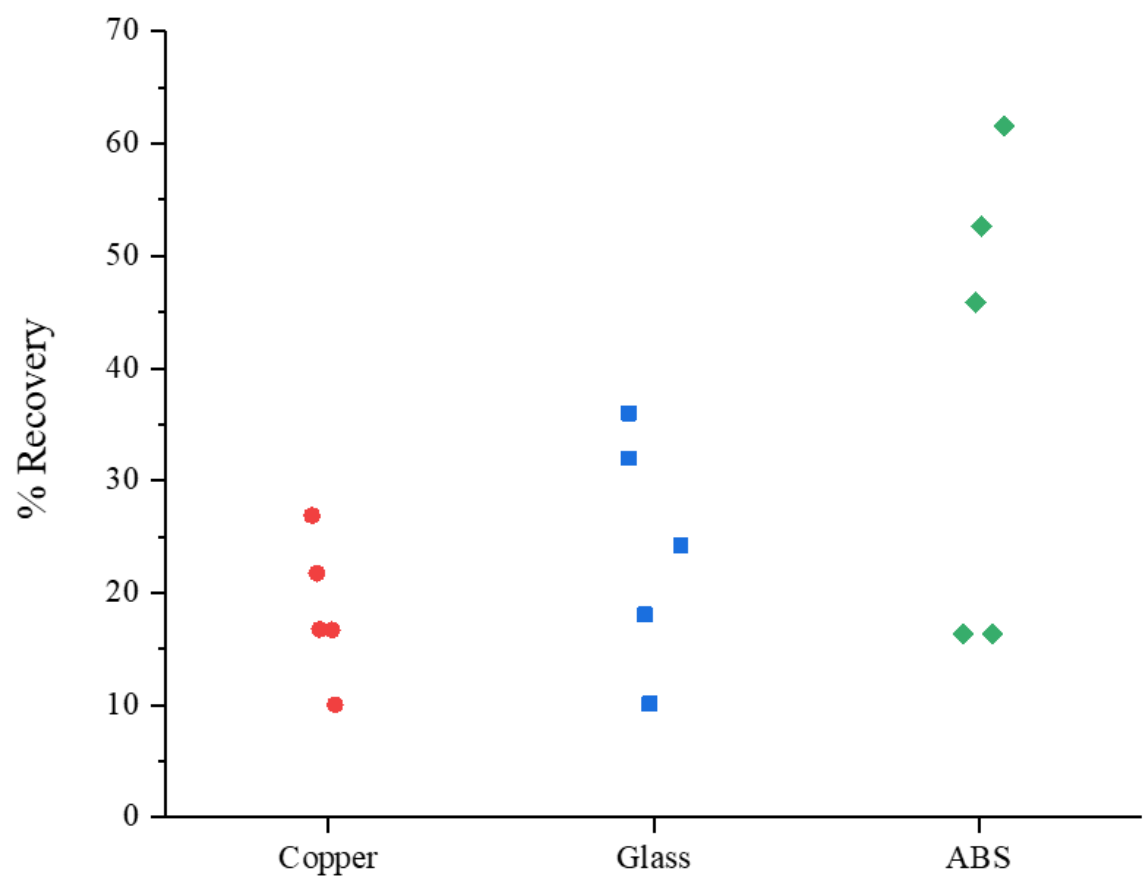

Fig. 4. Scatter plot of the recoveries (\%) obtained from the three non-porous surfaces tested. 


\subsection{All Surfaces}

The average recoveries obtained from each surface are visually displayed in Fig. 5. When comparing the recoveries obtained from porous and non-porous surfaces (Fig. 6), a significantly ( $<<0.05$ ) higher recovery was obtained from the non-porous test surfaces (average recovery of $27.0 \%$ ) than the porous test surfaces (average recovery $3.2 \%$ ). The recoveries of ammonium nitrate from porous surfaces yielded higher coefficients of variation $(\% \mathrm{CV}:$ wood $=68.2 \%$, fabric $=53.7 \%$, and carpet $=82.5 \%)$ than those from non-porous surfaces $(\% \mathrm{CV}$ : copper $=30.6 \%$, glass $=38.7 \%$, and $\mathrm{ABS}=48.8 \%)$. As such, a more consistent recovery was obtained when the gelatine-based collection medium was applied to non-porous surfaces.

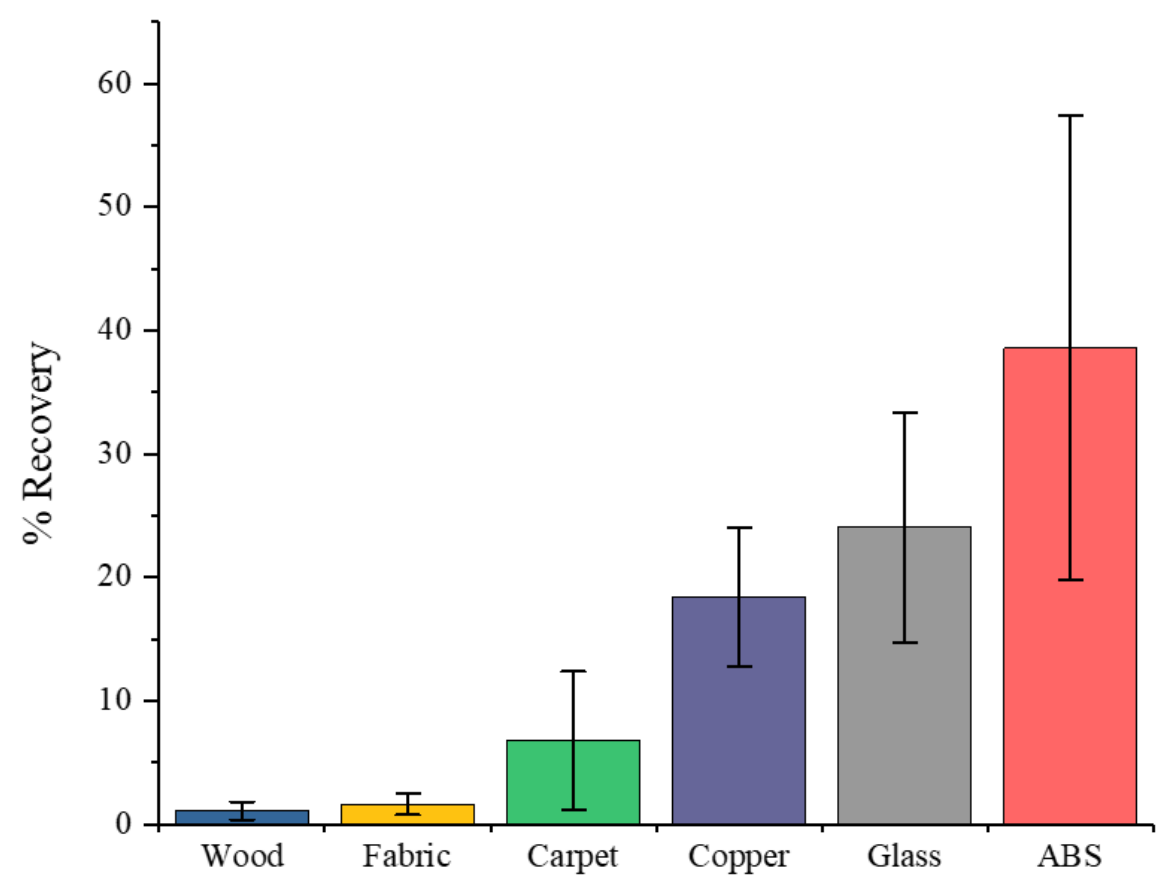

Fig. 5. Mean recoveries (\%) obtained from each surface. Error bars represent one standard deviation. 


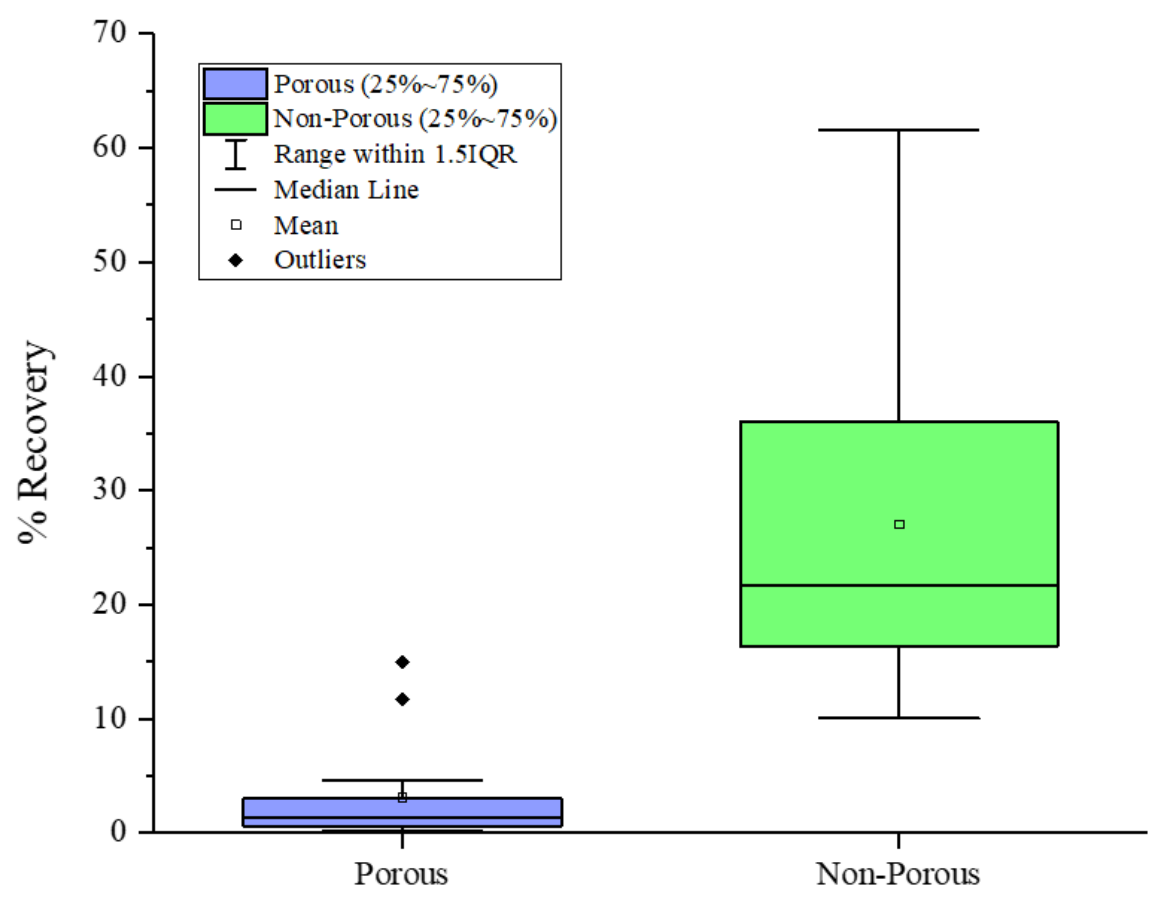

Fig. 6. Box plot comparing the recoveries $(\%)$ obtained from porous $(n=15)$ and non-porous surfaces $(n=15)$.

The gel-based formulation was able to recover ammonium nitrate from all three of the porous surfaces tested, although the recoveries were low. Previous published studies such as Yu et al. (2017), have highlighted the difficulties of recovering trace explosive compounds from porous surfaces [21]. With respect to the non-porous test surfaces, the recovery range obtained $(10-62 \%)$ was much higher and falls within the range of conventional swabbing methods $(10-90 \%)$ [18].

\subsection{Further Work}

This study presents the formulation and application of a gelatine-based recovery method for explosive residues, specifically ammonium nitrate. In order to ascertain the circumstances in which the application of a gelatine-based hydrogel might be more appropriate than other recovery methods, direct comparison studies are now required, accounting for larger sampling areas and a wide array of explosive compounds (both organic and inorganic). Additionally, the ability of the gel to recover trace quantities of these residues should be assessed in order to determine the amount of explosive that can be feasibly recovered using hydrogels. The application of hydrogels as a collection medium could be extended to other traces, such as drug and gunshot residues. Hydrogels may afford other advantages that require assessment, including: the long-term encapsulation of particulates during transfer and storage, the ability to be applied across complex surfaces, and the potential coverage across large surface areas. 


\section{Conclusion}

This study set out to create a gelatine-based medium and then assess the suitability of the gel for the collection and recovery of trace particulate explosive residues on a selection of common porous and non-porous surfaces. This study has demonstrated that:

1) it has been possible to create a low cost, effective gelatine-based formulation that is effective at recovering trace particulate explosive residues from a range of different surfaces;

2) using the gelatine-based formulation, ammonium nitrate was recovered from all of the test surfaces;

3) a significantly higher recovery rate was obtained from the non-porous surfaces tested than the porous surfaces;

4) the recovery rates across the test surfaces ranged from $0.1 \%$ to $61.6 \%$, with the highest recoveries obtained from $\mathrm{ABS}$ and the lowest from untreated wood.

The use of gel formulations could become a valuable asset in the forensic tool kit for the collection of explosive traces. A key attribute of the gel is that it offers an alternative recovery tool, with comparable recoveries from non-porous surfaces, to conventional swabbing and solvent extraction methods. As such, the results of this study provide a starting point from which further research can now be conducted. The method presented here offers an alternative collection approach that does not rely on the conventional swabbing materials and solvent systems. The results of this study are therefore promising and have the potential to offer a more effective method for inclusion in the toolkit of investigators tasked with the forensic collection and recovery of post-blast residues at bomb sites. 
CRediT authorship contribution statement

M.A. Amaral: Conceptualization, Formal analysis, Investigation, Methodology, Writing - original draft. S. Yasin: Formal analysis, Methodology, Writing - review \& editing. A.P. Gibson: Methodology, Project administration, Supervision, Visualization, Writing - review \& editing. R.M. Morgan: Funding acquisition, Methodology, Project administration, Supervision, Validation, Writing - review \& editing.

Declaration of competing interest

The authors declare that they have no known competing financial interests or personal relationships that could have appeared to influence the work reported in this paper.

This research did not receive any specific grant from funding agencies in the public, commercial, or not-for-profit sectors. 


\section{References}

[1] United States Department of Homeland Security

The 2014 Quadrennial Homeland Security Review

U.S. Department of Homeland Security, Washington, DC (2014), pp. 1-104

[2] J. Vermette

General Protocols at the Scene of an Explosion

In A. Beveridge (Ed.), Forensic Investigation of Explosions (2nd ed.)

CRC Press, Boca Raton, FL (2012), pp. 79-117

[3] N. Abdul-Karim, R. Morgan, R. Binions, T. Temple, K. Harrison

The Spatial Distribution of Postblast RDX Residue: Forensic Implications

Journal of Forensic Sciences, 58(2) (2013), pp. 365-371

DOI: $10.1111 / 1556-4029.12045$

[4] S. Bell

Forensic chemistry

Pearson, Upper Saddle River, NJ (2006), pp. 413-431

[5] J. Yinon, S. Zitrin

Modern Methods and Applications in Analysis of Explosives

John Wiley \& Sons, West Sussex, England (1996), pp. 163-209

[6] N. Abdul-Karim, C.S. Blackman, P.P. Gill, E.M.M. Wingstedt, B.A.P. Reif

Post-blast explosive residue - a review of formation and dispersion theories and experimental research

Royal Society of Chemistry (RSC) Advances, 4(97) (2014), pp. 54354-54371

DOI: $10.1039 / C 4 R A 04195 J$

[7] H.J. Yallop

Explosion Investigation

The Forensic Science Society, Harrogate, England (1980), pp. 13-28

[8] J. Akhavan

The Chemistry of Explosives (3rd ed.)

The Royal Society of Chemistry, Cambridge, England (2011), pp. 27-59

[9] R.B. Hopler

The History, Development, and Characteristics of Explosives and Propellants

In A. Beveridge (Ed.), Forensic Investigation of Explosions (2nd ed.)

CRC Press, Boca Raton, FL (2012), pp. 1-18 
[10] M. Bali

Niche Threat? Organic Peroxides as Terrorist Explosives

Australian Army Journal, 10(1) (2013), pp. 35-48

[11] A.D.D. Gordon

Muslims, Terrorism and Rise of The Hindu Right in India

Australian National University, Canberra, Australia (Working Paper No. 389) (2004)

[12] R. Ducibella, J. Cunningham

Design Considerations

In D.O. Dusenberry (Ed.), Handbook for Blast Resistant Design of Buildings

John Wiley \& Sons, Hoboken, NJ (2010), pp. 17-87

[13] W.E. Dyson

Terrorism: An Investigator's Handbook (4th ed.)

Elsevier, Waltham, MA (2011), pp. 1-18

[14] J.D. Kelleher

Explosives Residue: Origin and Distribution

Forensic Science Communications, 4(2) (2002)

[15] R.A Strobel

Recovery of Material from the Scene of an Explosion and Its Subsequent Forensic Laboratory Examination - A Team Approach

In A. Beveridge (Ed.), Forensic Investigation of Explosions (2nd ed.)

CRC Press, Boca Raton, FL (2012), pp. 119-157

[16] G.T. Murray

The Significance of Analytical Results in Explosives Investigation

In A. Beveridge (Ed.), Forensic Investigation of Explosions (2nd ed.)

CRC Press, Boca Raton, FL (2012), pp. 725-739

[17] C. Todd, L. Jones, M. Marshall

Explosions

In P. White (Ed.), Crime Scene to Court: The Essentials of Forensic Science (3rd ed.)

The Royal Society of Chemistry, Cambridge, England (2010), pp. 293-321

[18] D.A. DeTata, P.A. Collins, A.J. McKinley

A Comparison of Common Swabbing Materials for the Recovery of Organic and Inorganic Explosive Residues

Journal of Forensic Sciences, 58(3) (2013), pp. 757-763.

DOI: $10.1111 / 1556-4029.12078$ 
[19] N. Song-im, S. Benson, C. Lennard

Evaluation of different sampling media for their potential use as a combined swab for the collection of both organic and inorganic explosive residues

Forensic Science International, 222 (2012), pp. 102-110

DOI: 10.1016/j.forsciint.2012.05.006

[20] G. L. McEneff, A. Richardson, T. Webb, D. Wood., B. Murphy, R. Irlam, J. Mills, D. Green, L. P. Barron

Sorbent Film-Coated Passive Samplers for Explosives Vapour Detection Part B:

Deployment in Semi-Operational Environments and Alternative Applications

Scientific Reports, 8 (2018)

DOI: $10.1038 / \mathrm{s} 41598-018-24245-\mathrm{X}$

[21] H. A. Yu, T. Becker, N. Nic Daeid, S. W. Lewis

Fundamental studies of the adhesion of explosives to textile and non-textile surfaces

Forensic Science International, 273 (2017), pp. 88-95

DOI: 10.1016/j.forsciint.2017.02.008

[22] N. Song-im, S. Benson, C. Lennard

Establishing a universal swabbing and clean-up protocol for the combined recovery of organic and inorganic explosive residues

Forensic Science International, 223 (2012), pp. 136-147

DOI: 10.1016/j.forsciint.2012.08.017

[23] E.M. Ahmed

Hydrogel: Preparation, characterization, and applications: A review

Journal of Advanced Research, 6 (2015), pp. 105-121

DOI: $10.1016 /$ j.jare.2013.07.006

[24] A. Choodum, K. Malathong, N. NicDaeid, W. Limsakul, W. Wongniramaikul

A cost effective hydrogel test kit for pre and post blast trinitrotoluene

Forensic Science International, 266 (2016), pp. 202-208

DOI: $10.1016 /$ j.forsciint.2016.05.036

[25] W. Helmond, V. O’Brien, R. Jong, J. Esch, S. Oldenhof, M. Puit

Collection of amino acids and DNA from fingerprints using hydrogel

Analyst, 143 (2018), pp. 900-905

DOI: 10.1039/C7AN01692A 
O. Okay

\section{General Properties of Hydrogels}

In G. Gerlach \& K. F. Arndt (Eds.), Hydrogel Sensors and Actuators. Springer Series on Chemical Sensors and Biosensors (Methods and Applications)

Heidelberg, Berlin: Springer (2009), pp. 1-114

DOI: $10.1007 / 978-3-540-75645-3 \_1$

[27] K. Pal, A.K. Banthia, D.K. Majumdar

Polymeric Hydrogels: Characterization and Biomedical Applications - A mini review

Designed Monomers and Polymers, 12 (2009), pp. 197-220

DOI: $10.1163 / 156855509 X 436030$

[28] T. Miyata

Gels and Interpenetrating Polymer Networks

In N. Yuri (Ed.), Supramolecular Design for Biological Applications

CRC Press, Boca Raton, FL (2002), pp. 95-136

[29] A. Singh, P.K. Sharma, V.K. Garg, G. Garg

Hydrogels: A Review

International Journal of Pharmaceutical Sciences Review and Research, 4(2) (2010), pp. 97105

[30] A. R. Oppenheimer

IRA, the bombs and the bullets: a history of deadly ingenuity

Irish Academic Press, Dublin, Ireland (2008)

[31] M. Yücel, A. Bayrak, E. B. Yücel, C. S. Ozben

Simulations of Si-PIN photodiode based detectors for underground explosives enhanced by ammonium nitrate

Nuclear Instruments and Methods in Physics Research Section A: Accelerators, Spectrometers,

Detectors and Associated Equipment, 800 (2018), pp. 152-157

DOI: 10.1016/j.nima.2017.10.091

[32] United States Environmental Protection Agency

Method 8330B (SW-846): Nitroaromatics, Nitramines, and Nitrate Esters by High Performance Liquid Chromatography (HPLC)

U.S. Environmental Protection Agency, Washington, DC (2006), pp. 1-60 